\title{
PREVALENCE OF FLUOROQUINOLONE - RESISTANT \\ ENTEROBACTERIACEAE IN THE NORMAL RECTAL FLORA OF PATIENTS UNDERGOING TRANSRECTAL PROSTATE BIOPSY IN PHRAMONGKUTKLAO HOSPITAL, THAILAND
}

Vittaya Jiraanankul", Nopporn Choeypunt", Sudaluck Thunyaharn"*, Weelak Lerdpraiwan "

"Division of Urology, Department of Surgery, Phramongkutklao Hospital, Bangkok, Thailand

"Department of Microbiology, Faculty of Medical Technology, Nakhon Ratchasima College, Thailand

\begin{abstract}
Background: Despite routine use of antimicrobial prophylaxis, the incidence of infections after transrectal ultrasound-guided prostate biopsy has increased over the last 2 decades. Notably, a rising incidence of postbiopsy infections from fluoroquinolone-resistant Enterobacteriaceae had been reported over the same period. Many authors have studied the rectal flora reservoir to select the most appropriate prophylaxis antibiotics.

Objectives: The study aimed to determine the prevalence of fluoroquinolone-resistant and extended spectrum $\beta$-lactamase producing isolates at Phramongkutklao Hospital, Thailand and to identify the risk factors predicting the carriage of these organisms.

Methods: Men undergoing transrectal ultrasound-guided prostate biopsy were prospectively enrolled between February and October 2015. Rectal swab culture was obtained before antimicrobial prophylaxis and prostate biopsy. Univariate and multivariate analyses were performed to identify the independent risk factors associated with antimicrobial-resistant flora.

Results: In total, 99 patients underwent biopsy, of whom 38 (38.4\%) had antimicrobial-resistant rectal flora, with 26 (26.3\%) presenting fluoroquinolone-resistant rectal flora and $12(12.1 \%)$ having both fluoroquinolone-resistant rectal flora and extended spectrum $\beta$-lactamase. The incidence of postbiopsy infections was $6.1 \%$. The use of antibiotics in the past 6 months was found in $23.7 \%$ of the resistant group vs.6.6\% of the sensitive group (odds ratio $=4.86, p=$ 0.030 ), with the previous biopsy history being 31.6 and14.8\% (odds ratio $=3.17, p=0.036$ ), respectively. Postbiopsy infections occurred in 13.2 and $1 \%$ (odds ratio $=10.69, p=0.045$ ) of patients in the resistant and sensitive groups, respectively.

Conclusion: The prevalence of fluoroquinolone-resistant rectal flora increased among patients undergoing transrectal prostate biopsy at Phramongkutklao Hospital, Thailand. A history of antibiotics in the past 6 months, previous biopsy, and postbiopsy infections were associated with antimicrobial resistance. Culture-directed prophylaxis antibiotics may reduce postbiopsy infections after transrectal prostate biopsy
\end{abstract}

Keywords : Biopsy, Fluoroquinolone, Prostate, Risk factors, Thailand

J Southeast Asian Med Res 2018; 2(1): 1-6.

http://www.jseamed.org

Correspondence to:

Jiraanankul V, Division of Urology, Department of Surgery, Phramongkutklao Hospital, Bangkok, Thailand E-mail : zyrus118@hotmail.com 


\section{Introduction}

Transrectal ultrasound-guided prostate biopsy (TRUS-Bx) is the gold standard procedure for diagnosing prostate cancer, which is the most commonly diagnosed cancer and the second leading cause of cancer deaths among American men. In 2014, it has been estimated that 233,000 men would be diagnosed with prostate cancer and 29,480 would die of this disease. ${ }^{(1)}$ Approximately 800,000 biopsies are performed in the US alone annually. ${ }^{(2)}$ Although generally considered a safe procedure, complications can sometimes occur, including urinary tract infection, prostatitis, bacteremia, sepsis, hematuria, rectal bleeding, and hematospermia. ${ }^{(3)}$ In a larger series, the postbiopsy infection (PBI) rate ranged from 0.1 to $7 \% .{ }^{(4-6)}$ Clear evidence showed that prophylactic antimicrobials reduced the rates of PBIs after TRUS-Bx. ${ }^{(6)}$ Currently, the antimicrobial prophylaxis regimen, as recommended by the American Urological Association, is a single dose of fluoroquinolone(FQ) before TRUS-Bx, with cephalosporin or trimethoprim-sulphamethoxazole or aminoglycosides as alternatives. ${ }^{(7)}$

Despite routine use of antimicrobial prophylaxis, the incidence of PBI after TRUS-Bx has increased over the last two decades. ${ }^{(8-10)}$ Notably, a rising incidence of PBI from FQ-resistant Escherichia coli has been reported over the same period, leading many to conclude that the recent rise in infectious complications is related to FQ-resistance. ${ }^{(10-12)}$ Most PBIs arise from the direct inoculation of bacteria from the rectal mucosa into the urinary tract and prostatic vessels. Many authors have studied the rectal flora reservoir of patients undergoing TRUS-Bx to select the most appropriate prophylaxis antibiotics to reduce PBI. ${ }^{(13-18)}$

Based on this practice, the rates of rectal carriage have ranged from 10.6 to $22 \%$ for FQ-resistant flora in the US and Europe. ${ }^{(17,19-21)}$ In Asia, the prevalence of these organisms has ranged from 26.7 to $40.4 \% .{ }^{(22,23)}$ Studies of the risk factors associated with FQ-resistant bacteria have shown a relation to diabetes, history of antibiotic use in the last three months, hospitalization, previous prostate biopsy, indwelling of urinary catheter, and use of steroids. ${ }^{(17,19)}$ Our study aimed to determine the prevalence of $\mathrm{FQ}$ resistant rectal flora and extended spectrum $\beta$-lactamase (ESBL)-producing isolates at Phramongkutklao Hospital, Thailand, and to identify the risk factors predicting the carriage of these organisms.

\section{Methods}

We prospectively enrolled all male patients undergoing TRUS-Bx between February and October 2015 at Phramongkutklao Hospital, Thailand. The protocol for the research project was approved by the Institutional Review Board of the Royal Thai Army Medical Department. The exclusion criteria included active urinary tract or intestinal infection at the time of biopsy and coagulopathy.

Informed consent was obtained from eligible patients. Rectal swab culture was obtained before performing antimicrobial prophylaxis and prostate biopsy. For FQ-resistant testing, swabs were directly cultured on both MacConkey agar, with $1 \mathrm{mg} / \mathrm{L}$ ciprofloxacin, and blood agar plates and incubated at $37^{\circ} \mathrm{C}$ for $18 \mathrm{~h}$. Antimicrobial susceptibility testing was performed using the agar disk diffusion technique. All patients received a standard empirical prophylactic of FQ. Basic demographic and clinical data were collected from patients undergoing TRUS-Bx during the study period using questionnaires. The history of antibiotic use (less than six months), hospitalization, urethra catheter and previous prostate biopsy within six months was reviewed from medical records. The patients were followed for at least 30 days to check for infectious complications. Infections were defined as lower urinary tract symptoms with fever and positive urine culture ( $>104 \mathrm{cfu} / \mathrm{mL}$ ).

Descriptive statistics were used for the patients' demographic and background data. Univariate analyses were performed for variables that were possible predictors of PBIs using Pearson's chi-square test and Student's t-test. Variables were considered eligible for inclusion in a multivariate logistic regression model when they had a $\mathrm{p}$ value of $<0.05$.

\section{Results}

In total, 99 patients undergoing TRUS-Bx at Phramongkutklao Hospital between February and October 2015 were enrolled in the present study. The demographic data of the patients are shown in Table 1. Rectal swab culture showed that $94.9 \%(\mathrm{n}=94)$ involved Escherichia coli, followed by Klebsiella spp. $(4.1 \%, \mathrm{n}=4)$ and Enterococcus faecalis $(1 \%, \mathrm{n}=1)$. In total, $38.4 \% \quad(\mathrm{n}=38)$ of patients carried antimicrobial-resistant rectal flora, of whom $26.3 \%$ $(\mathrm{n}=26)$ had FQ-resistant rectal flora and $12.1 \%(\mathrm{n}=12)$ had both FQ-resistant rectal flora and ESBL. The incidence of PBI was $6.1 \%(n=6)$. The characteristics of the patients who developed PBI are shown in Table 2. 


\begin{tabular}{|c|c|c|}
\hline Parameters & Number & Percent \\
\hline \multicolumn{3}{|l|}{ Age } \\
\hline Mean \pm SD & & $67.25 \pm 8.07$ \\
\hline Range (Min-Max) & & $49-84$ \\
\hline \multicolumn{3}{|l|}{ PSA } \\
\hline Mean \pm SD & & $24.98 \pm 62.62$ \\
\hline Median & & 9.81 \\
\hline Range (Min-Max) & & $2.06-500$ \\
\hline \multicolumn{3}{|l|}{ Diabetes } \\
\hline Yes & 17 & 17.2 \\
\hline \multicolumn{3}{|c|}{ History of ATB use ( $<6$ months) } \\
\hline Fluoroquinolone & 10 & 10.1 \\
\hline Cephalosporin & 3 & 3.0 \\
\hline \multicolumn{3}{|l|}{ Past admission } \\
\hline Yes & 4 & 4.0 \\
\hline \multicolumn{3}{|c|}{ History of urethra catheter } \\
\hline Yes & 5 & 5.1 \\
\hline \multicolumn{3}{|c|}{ The frequency of TRUS-Bx } \\
\hline 1 & 78 & 78.8 \\
\hline 2 & 18 & 18.2 \\
\hline 3 & 3 & 3.0 \\
\hline \multicolumn{3}{|c|}{ The number of biopsy cores } \\
\hline 12 & 89 & 89.9 \\
\hline$>12$ & 10 & 10.1 \\
\hline
\end{tabular}

SD, standard deviation; PSA, prostate-specific antigen; ATB, antibiotic; TRUS-Bx, transrectal ultrasound-guided biopsy

Table 2 Characteristics of patients with postbiopsy infection

\begin{tabular}{|c|c|c|c|c|c|c|}
\hline \multirow{2}{*}{ Characteristics } & \multicolumn{6}{|c|}{ Postbiopsy patient } \\
\hline & 1 & 2 & 3 & 4 & 5 & 6 \\
\hline Age, $y$ & 55 & 68 & 67 & 56 & 68 & 73 \\
\hline PSA, ng/ml & 6.6 & 15 & 6.79 & 53 & 11.5 & 8.07 \\
\hline Rectal swab culture & E. coli & E. coli & E. coli & E. coli & E. coli & E. coli \\
\hline FQ-resistant & + & + & + & + & - & + \\
\hline ESBL & - & - & + & + & - & - \\
\hline Urine culture & Klebsilla & E. coli & E. coli ESBL & E. coli ESBL & E. faecalis & E. coli \\
\hline Blood culture & - & - & - & - & - & - \\
\hline
\end{tabular}

PSA, prostate-specific antigen; FQ, fluoroquinolone; ESBL, extend-spectrum $\beta$-lactamase producing 
FQ-resistance of a patient was not significantly the resistant group vs.6.6\% of the sensitive group [odds associated with age, mean PSA, DM, past admission, ratio $(\mathrm{OR})=4.86, p=0.030$ ], with a previous biopsy history urethra catheter and more than 12 core biopsies. FQ being 31.6 and14.8\% $(\mathrm{OR}=3.17, p=0.036)$, respectively. resistance flora significantly differed in recent antibiotic PBIs occurred in 13.2 and1\% $(\mathrm{OR}=10.69, p=0.045)$ of pause, previous prostate biopsy and PBIs between the two tients in the resistant and sensitive groups, respectively, groups revealed by the univariate analyses. The use of using multivariate logistic regression analysis. (Tables 3 antibiotics in the past six months was found in $23.7 \%$ of and 4).

Table 3 Univariate comparision of FQ resistance by clinical finding

\begin{tabular}{llll}
\hline & FQ-sensitive (\%) & FQ-resistant (\%) & $p$-value \\
\hline Mean age & 66.85 & 67.89 & 0.535 \\
Mean PSA & 23.31 & 27.65 & 0.739 \\
Diabetes & $8(13.1)$ & $9(23.7)$ & 0.650 \\
ATB use in 6 months & $4(6.6)$ & $9(23.7)$ & $0.023^{*}$ \\
Past admission & $1(1.6)$ & $3(7.9)$ & 0.187 \\
Urethra catheter & $3(4.9)$ & $2(5.3)$ & 0.311 \\
Previous prostate biopsy & $9(14.8)$ & $12(31.6)$ & $0.034^{*}$ \\
$>12$ cores biopsy & $7(11.5)$ & $3(7.9)$ & 0.667 \\
Postbiopsy infection & $1(1.6)$ & $5(13.2)$ & $0.019^{*}$
\end{tabular}

$* p<0.05$, PSA, prostate-specific antigen; ATB, antibiotics; FQ, fluoroquinolone

Table 4 Multivariate analysis of FQ resistance by clinical finding

\begin{tabular}{lll}
\hline & OR (95\% CI) & p-value \\
\hline Diabetes & 1.334 & 0.649 \\
& $(0.385-4.624)$ & $0.030^{*}$ \\
ATB use in 6 months & 4.860 & 0.208 \\
& $(1.170-20.192)$ & 0.330 \\
Past admission & 5.204 & $0.036^{*}$ \\
Urethra catheter & $(0.399-67.881)$ & 0.285 \\
& $(0.023-3.571)$ & 0.720 \\
Previous prostate biopsy & 3.173 & $(1.078-9.334)$ \\
& 0.720 & $0.045^{*}$
\end{tabular}

${ }^{*} p<0.05, \mathrm{FQ}$, fluoroquinolone; ATB, antibiotic; OR, odd ratios; 


\section{Discussion}

Currently, the antimicrobial prophylaxis regimen before TRUS-Bx is a single dose of FQ. ${ }^{(7)}$ In the present study, the incidence of PBI was $6.1 \%$; in related studies, the incidence of PBI ranged from 0.1 to $7 \%{ }^{(4-6)}$ The incidence of PBI has been increasing in recent years, along with the rising prevalence of FQ-resistant flora. The prevalence of FQ-resistant rectal flora was $38.4 \%$ in the present study. Based on these findings, we suggest that the use of FQ for prophylaxis before TRUS-Bx may not be effective at Phramongkutklao Hospital, Thailand. Alternative ways included adapting the prophylaxis antibiotics to local resistant rates or identifying patients carrying FQ-resistant organisms before the procedure using culturing rectal swabs.

In the present study, we showed that a history of previous TRUS-Bx and recent use of antibiotics were risk factors of FQ-resistant rectal flora. However, these risk factors may overlap among some patients because the patients undergoing biopsy had received the prophylaxis antibiotics too.

We conclude that when patients have an appointment for TRUS-Bx, the most effective method is to perform a rectal swab culture one to two weeks before the biopsy and select a prophylaxis antibiotic using the culture result to decrease the PBI rate. When the hospital is unable to perform a rectal swab culture, the history of the previous biopsy or antibiotic (FQ or cephalosporin) used in the past six months can be used to select the most appropriate antibiotic. The alternatives for prophylaxis antibiotics are aminoglycoside, carbapenem and fosfomycin.

In the present study, we found the following limitations. The first was the limited number of patients in the study; the study had a small sample size. The second was the patient data from hospital records may have underestimated the history of antibiotic use. The prevalence of fluoroquinoloneresistant rectal flora increased among patients undergoing TRUS-Bx at Phramongkutklao Hospital, Thailand. A history of antibiotics in the past six months, previous biopsy and PBIs were associated with antimicrobial resistance. Culturedirected prophylaxis antibiotics may reduce the infectious complications after TRUS-Bx.

\section{References}

1. Siegal R, Ma J, Zou Z, Jemal A. Cancer statistics, 2014. CA Cancer J Clin 2014; 64: 9-20.

2. Hodge KK, McNeal JE, Stamey TA. Ultrasound guided transrectal core biopsies of the palpably abnormal prostate. J Urol 1989; 142: 66-70.

3. Dai J, Leone A, Mermel L, Hwang K, Pareek G, Schiff $\mathrm{S}$. et al. Rectal swab culture-directed antimicrobial prophylaxis for prostate biopsy and risk of post procedure infection: a cohort study. Urology 2015; 85: 8-14.

4. Tal R, Livne PM, Lask DM, Baniel J. Empirical management of urinary tract infections complicating transrectal ultrasound guided prostate biopsy. J Urol 2003; 169: $1762-5$.

5. Feliciano J, Teper E, Ferrandino M, Macchia RJ, Blank $\mathrm{W}$, Grunberger I. et al. The incidence of fluoroquinolone resistant infections after prostate biopsy are fluoroquinolones still effective prophylaxis? J Urol 2008; 179: 952-5.

6. Kapoor DA, Klimberg IW, Malek GH, Wegenke JD, Cox CE, Patterson AL. et al. Single-dose oral ciprofloxacin versus placebo for prophylaxis during transrectal prostate biopsy. Urology1998; 52: 552-8.

7. Wolf JS Jr, Bennett CJ, Dmochowski RR, Hollenbeck BK, Pearle MS, Schaeffer AJ. Best practice policy statement on urologic surgery antimicrobial prophylaxis. J Urol 2008; 179: 1379-90.

8. Loeb S, Carter HB, Berndt SI, Ricker W, Schaeffer EM. Complications after prostate biopsy: data from SEERMedicare. J Urol 2011; 186: 1830-4.

9. Nam RK, Saskin R,Lee Y, Liu Y, Law C, Klotz LH. et al. Increasing hospital admission rates for urological complications after transrectal ultrasound guided prostate biopsy. J Urol 2013; 189 (1 suppl): S12-7.

10. Carignan A, Roussy JF, Lapointe V, Valiquette L, Sabbagh R, Pépin J. Increasing risk of infectious complications after transrectal ultrasound-guided prostate biopsies: time to reassess antimicrobial prophylaxis? Eur Urol 2012; 62: 453-9.

11. Williamson DA, Barrett LK, Rogers BA, Freeman JT, Hadway P, Paterson DL. Infectious complications following transrectal ultrasound-guided prostate biopsy: new challenges in the era of multidrug-resistant Escherichia coli. Clin Infect Dis 2013; 57: 267-74. 
12. Feliciano J, Teper E, Ferrandino M, Macchia RJ, Blank W, Grunberger I, et al. The incidence of fluoroquinolone resistant infections after prostate biopsye are fluo roquinolones still effective prophylaxis? J Urol 2008; 179: 952-5.

13. Batura D, Rao GG, Nielsen PB. Prevalence of antimicrobial resistance in intestinal flora of patients undergoing prostatic biopsy: implications for prophylaxis and treatment of infections after biopsy. BJU Int 2010; 106: 1017-20.

14. Minamida S, Satoh T, Tabata K, Kimura M, Tsumura H, Kurosaka S. et al. Prevalence of fluoroquinolone-resistant Escherichia coli before and incidence of acute bacterial prostatitis after prostate biopsy. Urology 2011; 78: 1235-9.

15. Taylor S, Margolick J, Abughosh Z, Goldenberg SL, Lange D, Bowie WR, et al. Ciprofloxacin resistant in the faecal carriage of patients undergoing transrectal ultrasound guided prostate biopsy. BJU Int 2013; 111: 946-53.

16. Duplessis CA, Bavaro M, Simons MP, Marguet C, Santomauro M, Auge B. et al. Rectal cultures before transrectal ultrasound-guided prostate biopsy reduce post-prostatic biopsy infection rates. Urology 2012; 79: 556-61.

17. Steensels D, Slabbaert K, De Wever L, VermeerschP, Van Poppel H, Verhaegen J. Fluoroquinolone resistant E. coli in intestinal flora of patients undergoing transrectal ultrasound-guided prostate biopsy--should we reassess our practices for antibiotic prophylaxis? Clin Microbiol Infect 2012; 18: 575-81.

18. Taylor AK, Zembower TR, Nadler RB, Scheetz MH, Cashy JP, Bowen D. et al. Targeted antimicrobial prophylaxis using rectal swab cultures in men undergoing transrectal ultrasound guided prostate biopsy is associated with reduced incidence of postoperative infectious complications and cost of care. J Urol 2012; 187: 1275-9.

19. Batura D, Rao GG, Nielsen PB. Prevalence of antimicrobial resistant in intestinal flora of patients undergoing prostatic biopsy: implications for prophylaxis and treatment of infections after biopsy. BJU Int 2010; 106: 1017-20.

20. Qi C, Malczynski M, Schaeffer AJ, Barajas G, Nadler $\mathrm{RB}$, Scheetz MH. et al. Characterization of ciprofloxacin resistant Escherichia coli isolates among men undergoing evaluation for transrectal ultrasound guided prostate biopsy. J Urol 2013; 190: 2026-32.

21. Duplessis CA, Bavaro M, Simons MP, Marguet C, Santomauro M, Auge B. et al. Rectal cultures before transrectal ultrasound-guided prostate biopsy reduce post-prostatic biopsy infection rates. Urology 2012; 79: 556-61.

22. Lee JW, Park SC, Kim MK, Cheon MW, Kim GY,Cho $\mathrm{JH}$. Prevalence of antimicrobial resistant in normal rectal flora of patients undergoing transrectal ultrasonography-guided prostate biopsy in Korea. Int J Urol 2014; 21 : 811-4.

23. Tsu JH, Ma WK, Chan WK, Lam BH, To KC, To WK. et al. Prevalence and predictive factors of harboring fluoroquinolone-resistant and extended-spectrum B-lactamase-producing rectal flora in Hong Kong Chinese men undergoing transrectal ultrasound-guided prostate biopsy. Urology 2015; 85: 15-22. 